\title{
NOTE ON SEPARABILITY*
}

BY R. G. PUTNAM

The following theorems have been shown by R. L. Moore $\dagger$ to hold in a class $D$ of Fréchet.

THEOREM 1. In order that every subclass of a given class $D$ of Frêchet should be separable, it is necessary and sufficient that every uncountable subclass of that class $D$ should have a limit point.

Theorem 2. If $D_{s}$ is a separable class $D$, then every uncountable subclass of $D_{s}$ contains a point of condensation.

TheOREM 3. Every subclass of a separable class $D$ is itself separable.

THEOREM 4. In order that every uncountable subclass of a given class $D$ should contain a point of condensation of itself, it is necessary and sufficient that every uncountable subclass of $D$ should have a limit point.

THEOREM 5. In order that every ascending sequence of distinct closed subsets of a given class $D$ should be countable, it is necessary and sufficient that every descending one should be.

Theorems 3 and 4 follow from Theorems 1 and 2, and 5 is obtained with the aid of Theorems 1 and 4 .

* Presented to the Society, September 6, 1928.

† Fundamenta Mathematicae, vol. 8, p. 189. Theorems 1, 2, and 3 have been previously considered by W. Gross in Zur Theorie der Mengen, in denen ein Distanzbegriff definiert ist, Sitzungsberichte, Wien, vol. 123 (1914), pp. 801-819. See also a reference to this article in $A n$ acknowledgement, by R. L. Moore, Fundamenta Mathematicae, vol. 8, p. 374.

$\ddagger$ A class $D$ of Fréchet is a class of elements which satisfy the following conditions:

1. With every pair of elements $A$ and $B$ there is associated a number $(A, B)=(B, A) \geqq 0$.

2. $(A, B)=0$ if, and only if, $A=B$.

3. If $A, B$ and $C$ are any three elements, then $(A, C) \leqq(A, B)+(B, C)$.

4. The sequence of elements $P_{1}, P_{2}, P_{3}, \cdots$ converges to a limit $P$ if and only if the distance $\left(P, P_{n}\right)$ approaches zero as $n$ becomes infinite. A class in which conditions 1, 2 and 4 hold but in which 3 need not hold is a class $E$. 
The following example, due to P. Alexandroff, shows that none of the first four theorems stated above is true without change in all classes $E$. Consider the space composed of two parallel lines $A$ and $B$. Let the écart of any two distinct points $x$ and $y$ both of which are on $A$ or on $B$ be unity while the écart of a point $x$ on $A$ and a point $y$ on $B$ is the ordinary distance from $x$ to the projection of $y$ on $A$ or vice versa; if $x$ and $y$ are not distinct their écart is zero. In this space the limit points of a set entirely on one line are all on the other line. The space thus formed is a separable class $E$. Every uncountable subclass has a limit point but is not always separable and does not contain a point of condensation. Theorem 4 is not true in this space.

Theorems $1^{\prime}, 2^{\prime}, 4^{\prime}$, and $5^{\prime}$ which follow, are modifications respectively of Theorems $1,2,4$, and 5 , and can be shown to hold in classes $E$ (not at the same time classes $D$ ).

THEOREM $1^{\prime}$. In order that every subclass of a given class $E$ should be separable, it is necessary and sufficient that every uncountable subclass of that class $E$ should contain a limit point of itself.

The proof of this theorem is the same as the proof of Theorem 1 in Professor Moore's paper.

TheORem 2'. If every subclass of a given class $E$ is separable, then every uncountable subclass of that class $E$ contains a point of condensation of itself.

The proof of this theorem follows from the first part of the proof of Theorem 2.

Theorem 3 does not hold in all classes $E$ as the above example shows.

THEOREM $4^{\prime}$. In order that every uncountable subclass of a given class $E$ should contain a point of condensation of itself, it is necessary and sufficient that every uncountable subclass of the given class should contain a limit point of itself.

This theorem follows at once from Theorems $1^{\prime}$ and $2^{\prime}$.

THEOREM 5'. In order that every ascending sequence of distinct closed subsets of a given class $E$ in which every derived set is closed should be countable, it is necessary and sufficient that every descending one should be. 
This theorem is a consequence of Theorems $1^{\prime}$ and $4^{\prime}$ and the result of Sierpinski, used by Professor Moore in the proof of Theorem 5.

New York University

\section{NOTE ON A SCHOLIUM OF BAYES}

BY F. H. MURRAY

In his fundamental paper on a posteriori probability,* Bayes considered a certain event $M$ having an unknown probability $p$ of its occurring in a single trial. In deriving his a posteriori formula he assumed that all values of $p$ are equally likely, and he recommended this assumption for similar problems in which nothing is known concerning $p$. In the corollary to proposition 8 he derives the value

$$
\int_{0}^{1}\left(\begin{array}{l}
n \\
x
\end{array}\right) p^{x}(1-p)^{n-x} d p=\frac{1}{n+1}
$$

for the probability of $x$ successes in $n$ trials. This result is independent of $x$; in a scholium he observes that this consequence is what is to be expected, on common sense grounds, from complete ignorance concerning $p$, and this concordance is considered to justify the assumption that all values of $p$ are equally likely. $\dagger$

In order to complete the argument of the scholium it is necessary to show that no other frequency distribution for $p$ has the same property.

More precisely, given that a cumulative frequency function $f(p)$ has the property that for $0 \leqq x \leqq n, x, n$ being integers,

$$
\int_{0}^{1}\left(\begin{array}{l}
n \\
x
\end{array}\right) p^{x}(1-p)^{n-x} d f(p)=\frac{1}{n+1}
$$

* Bayes, An essay towards solving a problem in the doctrine of chances, Philosophical Transactions of the Royal Society, vol. 53 (1763), pp. 370-418.

$\dagger$ In other words, the assumption "all values of $p$ are equally likely" is equivalent to the assumption "any number $x$ of successes in $n$ trials is just as likely as any other number $y, x \leqq n, y \leqq n$." It has been suggested verbally by $\mathrm{Mr}$. E. C. Molina that this proposition has a possible importance in certain statistical questions. 